\title{
Elementos de apoyo a la gestión de inventario en las empresas ferreteras del municipio Lagunillas
}

\author{
Elements of support to the inventory management in the ferreteras \\ companies of the Lagunillas municipality
}

RESUMEN La investigación se centró en analizar los elementos de apoyo a la gestión de inventario presentes en las empresas ferreteras del municipio lagunillas. Metodologicamente se consideró descriptiva, con diseño de campo, no experimental y transeccional. La población estuvo constituida por las siete (7) empresas ferreteras del municipio Lagunillas, siendo los 33 sujetos informantes entre gerentes y administradores. La información se recolectó con un cuestionario contentivo de 18 ítems, con escala de frecuencia. El mismo fue validado por cinco (5) expertos, y la confiabilidad calculada por la formula Alfa de Cronbach cuyo coeficiente fue de 0,86 . Se concluye que, los elementos de apoyo a la gestión de inventario es una fortaleza para las empresas analizadas, en cuanto a infraestructura, talento humano, proveedor, innovación tecnológica, delegación de responsabilidades, plazo de entrega, disponibilidad y transporte. No obstante, se evidencian oportunidades de mejora en la capacitación del personal e innovación tecnológica.

Palabras clave Gestión de inventario, elementos de apoyo, talento, infraestructura, proveedores.

\footnotetext{
ABSTRACT $\mid$ The research focused on analyzing the elements of inventory management support present in the hardware companies of the Lagunillas municipality. Methodologically it was considered descriptive, with a field design, not experimental and transectional. The population consisted of the seven (7) hardware companies in the Lagunillas municipality, with the 33 reporting subjects being managers and administrators. The information was collected with a questionnaire containing 18 items, with a frequency scale. It was validated by five (5) experts, and the reliability calculated by Cronbach's Alpha formula, whose coefficient was 0.86 . It is concluded that the inventory management support elements is a strength for the analyzed companies, in terms of infrastructure, human talent, supplier, technological innovation, delegation of responsibilities, delivery time, availability and transportation. However, opportunities for improvement in personnel training and technological innovation are evident.

Keywords Inventory management, support elements, talent, infrastructure, suppliers
} 
INTRODUCCIÓN

El entorno económico tiende a cambiar con el pasar del tiempo, por ello todas las cosas que rodean a las empresas a nivel mundial, afectan de manera directa o indirecta su funcionamiento, en reacción a esto las empresas se ven en la necesidad de acoplarse a las exigencias de un mundo globalizado, generando como consecuencia que sus necesidades sean cada vez mayores. Por ello, las organizaciones para mantenerse activas dentro del entorno empresarial, deben ofrecer productos y servicios de calidad, ajustándose a los requerimientos y exigencias del mercado, siendo clave que sus procesos se ejecuten sobre la base de criterios de calidad, orientando sus actividades hacia la búsqueda de la eficiencia, racionalidad de sus recursos, así como el logro de ventajas competitivas.

Al respecto, el manejo de inventario se ha representado en las empresas como un punto álgido de gerencia empresarial, debido a que es un componente fundamental de la productividad, evidenciándose que en mercados muy competitivos, las compañías deben trabajar con inventarios cada vez más bajos y con niveles de servicios cada vez más altos. En esta perspectiva, la gestión de inventario es un elemento determinante para el éxito de la organización, dado que permitirá el uso racional de los recursos, logrando de esta forma la continuidad de los procesos, mediante la adquisición de materiales ajustados a los requerimientos de las funciones principales.

Por ello, la entrada, procesamiento y salida de materia prima, es el objeto fundamental de análisis de la gestión de inventario, así como la adquisición, procesamiento, inclusive la evaluación del insumo primario, buscando el logro en la ejecución de actividades específicas del proceso productivo, de acuerdo a los parámetros establecidos en el proceso de planificación de las empresas.

De acuerdo a lo anterior, para Render y otros. (2006) la gestión de inventario implica la existencia de todos los recursos utilizados dentro de la organización para desarrollar sus actividades y darle continuidad a cada uno de los procesos, donde el sistema de inventarios, es un conjunto de políticas y controles para supervisar los niveles, determinando cuáles son los idóneos para mantenerse, cuando sea necesario reabastecer el inventario y el tamaño ideal de los pedidos.

Según Anaya (2014), define la gestión de inventario como el mantenimiento de cantidades suficientes de bienes (refacciones y materias primas) que garanticen operaciones fluidas en sistemas de producción 0 actividades comerciales. Esta gestión constituye el objetivo fundamental dentro de la cadena de suministro, debido a que el nivel puede llegar a suponer la mayor inversión de la compañía, abarcando incluso más del $50 \%$ del total de activo en el sector de la distribución.

Para Ballou (2004), la gestión de inventario está constituida por bienes de la entidad destinados a la venta o producción para su posterior comercio, tales como son materia prima, producción en proceso, artículos terminados y otros materiales utilizados como empaque, envase de mercancía o refacciones para el mantenimiento y se consumen en el ciclo de operaciones. 
En el caso venezolano, se resalta lo planteado por Marcano (2011), para quien en la actualidad muchas organizaciones comerciales en Venezuela han reaccionado a la realidad de que al mejorar sus inventarios mejoran todos sus movimientos económicos, tanto internos como externos, y se encuentran en la búsqueda de soluciones para satisfacer sus necesidades, desde las más simples hasta las más complejas, además, de las de sus clientes, en términos de cualquier movimiento que esta pueda realizar.

Desde estas perspectivas, a criterio del investigador, en la actualidad, los elementos de apoyo a la gestión de inventario ha evolucionado hacia una forma de control integrado, incorporando la totalidad de funciones y recursos humanos en la actividad empresarial, desde el ultimo operario hasta el primer gerente, pues cada nivel dentro de la estructura organizacional tiene su control específico y definido en el que se ha integrado consecutivamente hasta llegar al nivel más alto.

En esta perspectiva, Render y otros (2006) consideran que es vital para toda empresa, industria o comercio llevar inventarios confiables, ya que esto garantizará una mayor seguridad en el proceso diario de movimiento de productos dentro de los almacenes. Así entonces, según los autores citados, existen varios elementos de apoyo a la gestión de inventario, entre los cuales destacan: infraestructura, talento humano, proveedor, capacitación del personal, innovación tecnológica, delegación de responsabilidades, entrega, disponibilidad y transporte.

En este orden de ideas, para Ballou (2004), los elementos de apoyo a la gestión de inventario permiten a la empresa decidir los medios a necesitar para sus operaciones y distribuir esos medios fabricar el producto deseado en las cantidades requeridas, en el menor tiempo y costo posible. Estos elementos facilitan el logro de los objetivos de la gestión de inventario, dirigidos a: disponer de materias primas y demás elementos de fabricación en el momento oportuno y lugar requerido; reducir los períodos muertos de maquinaria y obreros; asegurar que estos no trabajan en exceso, ni estén inactivos. Incluye esta autora, como elementos de apoyo a la gestión de inventario los siguientes: infraestructura, talento humano, proveedor, capacitación del personal, innovación tecnológica, delegación de responsabilidades, entrega, disponibilidad y transporte.

A fines de la investigación, los elementos de apoyo a la gestión de inventario son todos los elementos presentes en la organización que le permiten establecer sus debilidades y fortalezas para el desarrollo de cualquiera de las gestiones realizadas, en este caso la de inventario. Todos estos elementos se interrelacionan con una logística coordinada, encargada de minimizar los recursos financieros invertidos en la gestión de inventario. En este sentido, se considerarán los planteados por Render y col. (2006) y Ballou (2004), dado que se ajustan a las empresas del sector bajo estudio, por lo cual se asumen como indicadores para medir esta dimensión los elementos relacionados a: infraestructura, talento humano, proveedor, capacitación del personal, innovación tecnológica, delegación de responsabilidades, entrega, disponibilidad y transporte. A continuación, se abordan cada uno de ellos al detalle.

Según Fernández (2010), la infraestructura es el conjunto de estructuras de ingeniería e instalaciones, generalmente de larga vida útil, 
que constituyen la base sobre la cual se realiza la producción o la prestación de servicios necesarios para el desarrollo de fines productivos. Así, la infraestructura permite la asignación y realización coordinada de las tareas que conducen a las metas organizacionales, de tal forma, las distintas funciones de planificación, información, gestión, coordinación, presupuesto, sean responsabilidad de un sistema determinado.

Con base a lo reseñado por el autor citado, el investigador comprende que, la infraestructura es considerada como el sistema de instalaciones, equipos y servicios necesarios para el funcionamiento de una organización. Como elemento de apoyo a una efectiva gestión de procura, esta debe estar compuesta por edificios, estructuras de ingeniería, almacenes, zonas de carga y descarga, estacionamientos, espacio de trabajo e instalaciones adecuadas para el normal funcionamiento de las actividades, así como también, redes de telecomunicaciones, energía, abastecimiento de agua potable, saneamiento, transporte, entre otros, considerados muy necesarios para el desarrollo eficaz de los sistemas productivos.

En torno al talento humano, Alles (2005) lo define como el concepto de los conocimientos que las personas poseen o desarrollan a partir del despliegue de la calidad de las relaciones que se mantienen con miembros y equipos pertenecientes a la organización. El talento humano es un capital o un activo intangible que explican buena parte de la valoración que la sociedad y comunidad científica conceden a una organización. Como complemento, para Ivancevich y col. (2010), el talento humano es un activo intangible el cual se revaloriza mientras más se usa; así, se dice que la información con el añadido de la inteligencia se transforma en conocimiento, y el conocimiento con el añadido de la imaginación se convierte en innovación.

En este orden de ideas, el investigador asume que el talento humano representa para la gestión de inventario el elemento garantizador de la solidez en las pericias técnicas, un alto nivel de especialización, la experiencia y vocación de servicio, atención al cliente, el realizador de todos los procesos relacionados al inventario; por ello el personal de la empresa debe ser consciente del rol social y estratégico bajo su responsabilidad dentro de la organización, pues el mismo es la garantía de la excelencia que caracterizará a las empresas en cada una sus actividades.

Por otra parte, los proveedores son personas o entidades encargadas de suministrar materias primas, servicios y repuestos, entre otros, necesarios para que la empresa pueda desarrollar su actividad normalmente. Son los encargados de mantener viva y activa la organización y, por lo tanto, su importancia radica en el papel que desempeñan en la existencia y en el futuro de la empresa, de acuerdo a como lo afirma (Ballou, 2004).

A criterio del investigador, los proveedores representan a la organización, persona o empresa, que está en capacidad de suministrar los bienes o servicios requeridos para ejecutar el proceso productivo o la prestación del servicio. Normalmente este elemento de apoyo a la gestión de inventario se realiza en el departamento de compras. En este sentido, se debe considerar que llegar a construir un listado confiable, eficiente y responsable de proveedores resulta costoso en términos de tiempo y dinero 
para las organizaciones, por lo cual, se recomienda tener un registro de proveedores reducido.

De igual forma, la capacitación es un proceso continuo, porque aun cuando al personal de nuevo ingreso se le dé la inducción en forma adecuada, con frecuencia es preciso entrenarlos o capacitarlos en las labores para las que fueron contratados $y / 0$ proporcionales nuevos conocimientos necesarios para el desempeño de un puesto; al igual, los empleados con experiencia que son ubicados en nuevos puestos, pueden requerir capacitación para desempeñar adecuadamente su trabajo, según lo afirman (Werther y otros, 2008).

Las razones para proporcionar capacitación pueden ser muy variadas, según el criterio de Fernández (2010), y entre sus beneficios se encuentran: ayuda a mejorar las aptitudes y las actitudes; eleva los conocimientos de los ocupantes de los puestos en todos los niveles organizacionales; mejora la moral y la satisfacción de la fuerza de trabajo; guía al personal a identificarse con los objetivos de la empresa; crea una mejor imagen tanto del personal como de la organización; fluyen mejor la toma de decisiones y la solución de problemas; propicia el desarrollo y promociones; contribuye a mantener bajos los costos de operación en muchas áreas; contribuye positivamente en el manejo de conflictos y tensiones; permite el establecimiento y logro de metas individuales.

En este sentido, para el investigador, la capacitación es un elemento muy importante y representa un papel preponderante en la vida de las organizaciones y del personal de las mismas, así, puede decirse, aunque la capacitación o el entrenamiento auxilia a los miembros de la organización a desempeñar su trabajo actual, sus beneficios pueden prolongarse a toda su vida laboral y pueden auxiliar en el desarrollo de esa persona para cumplir futuras responsabilidades.

Así mismo, la innovación es la transformación de una idea en algo vendido o usado, esta es la materia prima, a partir de la cual se consiguen las innovaciones, según la definen Escorsa y Valls (2005). Dicen estos autores, sin ideas, no hay innovación posible, pues ella se convierte en un tema realmente importante, las mismas deben ser de alta calidad, para generar nuevos productos, resolver problemas y tomar decisiones. Uno de los aspectos más importantes es la originalidad, estas ideas no deben proceder del exterior, deben generarse en el interior de la mente humana, apareciendo la creatividad.

Mientras, Ivancevich y otros. (2010 p.84) definen la innovación tecnológica como el "conjunto de actividades mediante las cuales se traspasa el conocimiento tecnológico a una realidad física y pueda utilizarse con toda su velocidad". De allí, las innovaciones tecnológicas dan lugar a la modificación de la información, toma de decisiones y el proceso de organización, planificación del trabajo, a tal punto, que las empresas serán más proveedoras de servicios/productos, obteniendo como resultado una ventaja competitiva, ampliándose a un ritmo más rápido en términos de calidad y dimensión.

Desde la óptica del investigador, la innovación tecnológica es un factor relevante en el desarrollo de la gestión de inventario, pues la misma se basa en la eficiencia (gestión de recursos) y eficacia (consecución de objetivos), mediante la utilización de estrategias y ejecución 
de proyectos que contribuyan a ofrecer un mejor servicio al cliente mediante el crecimiento empresarial.

En lo que se refiere a la delegación de responsabilidades, es una de las aptitudes fundamentales que todo gerente debe poseer, considerando como la delegación sirve de medio para obtener mejores resultados, gracias a que evita la concentración de tareas en una sola persona y brinda al jefe la posibilidad de concentrarse en los temas prioritarios de su función, dejando a otros la ejecución de las tareas de carácter más operativo, según lo asegura Alles (2007). Asimismo, sirve para motivar a los colaboradores, pues implica depositar confianza en las capacidades del otro, en la creencia de ser capaz de realizar con éxito la tarea encomendada, brindándole la oportunidad de mostrar sus habilidades en el desarrollo de actividades no incluidas en sus tareas cotidianas.

Sobre la base de la idea expuesta, el investigador infiere que, la delegación de responsabilidades es factor relevante de cualquier gestión, pues permite a la empresa ahorrar tiempo y procesos, debido a que el directivo puede dedicarse a otras funciones. Para los empleados, incrementa la participación, estimula las iniciativas, motiva al personal, permitiendo a éste involucrarse en otras áreas de la empresa y desarrollar su sentido de pertenencia a la organización.

De la misma forma, el plazo de entrega representa el tiempo transcurrido desde el lanzamiento de la orden de compra hasta la recepción del material, así lo afirma García (2008). El lanzamiento de órdenes suele generarse de sistemas como el Materials Requirements Planning o planificación de las necesidades de materiales, aunque también responde a las políticas de gestión de inventario mantenidas en la empresa. Un objetivo primario del departamento de compras, en empresas de fabricación, es la entrega a tiempo de materiales de calidad adecuada para su uso en producción (o en distribución).

A fines de la investigación, el plazo de entrega es el lapso establecido por el proveedor para entregar el material en las propias instalaciones $\mathrm{o}$ en un sitio autorizado de recepción de la empresa, contado a partir de la colocación en firme del pedido.

Por otro parte, el principal objetivo del manejo del inventario debe ser asegurar que el producto esté disponible en el momento y en las cantidades deseadas, según Ballou (2004). De igual forma, para Vollmann y otros. (2008), implica tener un inventario que cumpla de manera regular con los requerimientos de materiales o productos del cliente. Al respecto afirma Escudero (2011), la tecnología de la información facilita la flexibilidad del sistema y proporciona nuevos modos para alcanzar una alta disponibilidad hacia los clientes, donde estos no representen una elevada inversión de capital en el inventario. La información a facilitar la flexibilidad, con respecto a la disponibilidad del inventario, es fundamental para lograr un desempeño logístico adecuado.

En este punto, el investigador asume, la disponibilidad es un factor relevante en cuanto se relaciona con la satisfacción del cliente, la misma consiste en la verificación de existencias o posibilidad de ubicar los bienes requeridos en los diferentes almacenes de la empresa, así como ofrecer sustitutos. Cabe destacar entonces, la disponibilidad de los materiales está relacionada 
al tiempo de entrega y cumplimiento por parte de los proveedores, donde en ocasiones se presentan inconvenientes al momento de la entrega trayendo como resultado inconformidad por parte de los usuarios.

Por último, el transporte es una actividad fundamental de la logística, asociada como un elemento de apoyo a la gestión de inventario, de acuerdo al criterio de Bowersox y otros. (2007), la cual consiste en colocar los productos de importancia en el momento preciso y en el destino deseado. El movimiento de materias primas y personas se realiza mediante las denominadas redes de transporte. Una red, es un conjunto de rutas conectadas por nodos, construidas para ser posibles los movimientos de mercaderías y servicios.

En relación a esto, el investigador asume el transporte como elemento fundamental de la gestión logística, y de apoyo a la de inventario, el cual permite la movilización, el flujo y manejo de los recursos a ser almacenados y distribuidos hasta su destino final Esto permite un ahorro en costos así como en horas-hombre inherentes al proceso de producción. Por tanto, cuando este elemento emerge como actividad de apoyo a la gestión de inventario su participación es importante, ocupándose del movimiento físico.

Por lo anteriormente expuesto, en el contexto de las empresas comerciales, éstas deben considerar que existe un mercado competitivo cada vez más dinámico, lleno de oportunidades, pero también de amenazas, las cuales surgen a cada momento provenientes del entorno, esto exige de la preparación necesaria para enfrentar a sus competidores, así como, conocer si cuentan con una gestión efectiva en el manejo de sus inventarios que les permita desafiar los retos y riesgos presentados en situaciones determinadas.

A estas situaciones no escapan las pequeñas y medianas empresas ferreteras, cuya naturaleza es ofrecer atención personalizada a las grandes empresas industriales, consumidores y población en general, a través de un gran surtido de productos propios del ramo; cuyo objetivo principal puede ser la compra, venta, distribución, importación y exportación al mayor y al detal de toda clase de materiales para la construcción. Ahora bien, a fines específicos del objeto de estudio, muchas de las pequeñas y grandes empresas ferreteras, que tienen su domicilio fiscal en el municipio Lagunillas del estado Zulia, presentan serios problemas en cuanto su gestión de inventario, afirmación que obedece a entrevistas informales realizadas por el investigador al personal que labora en las mismas, donde se pudo evidenciar, entre varios aspectos:

En muchas ocasiones los productos no se hayan almacenados correctamente en los estantes, existiendo productos mezclados con otros, aun cuando pertenecen a diferentes categorías, siendo en ocasiones una de las causas el exceso de productos adquiridos adicionalmente, lo cual muestra que no existe un control efectivo para la adquisición y almacenamiento. De igual manera, se pudo conocer que en ocasiones se presentan devoluciones de productos, a causa de que éstos no presentan buenas condiciones para ser utilizados por el cliente, la causa del inventario no está siendo almacenado en lugares óptimos de acuerdo a su característica, sufriendo así deterioro y trayendo como consecuencia merma en la inversión. 
Con base en las consideraciones presentadas, tomando en cuenta que las organizaciones en general, y las empresas del sector ferretero en lo particular, deben dar respuesta satisfactoria a sus clientes mediante una adecuada gestión que incluye el inventario como garante de la eficiencia operacional, se realizó esta investigación con el propósito de analizar los elementos de apoyo a la gestión de inventario presentes en las empresas ferreteras del municipio lagunillas

MATERIALES Y METODO

En este sentido, y de acuerdo a la problemática planteada referida empresas ferreteras del municipio Lagunillas, el estudio se clasificó como descriptivo, con un diseño de campo, no experimental, y transeccional. La población fueron las siete (07) pequeñas y medianas empresas ferreteras del municipio Lagunillas, que cumplieran con el criterio de inclusión de tener más de 15 años registradas y activas en el SENIAT (Servicio Nacional Integrado de Administración Aduanera y Tributaria), y a su vez, fueran miembros de la Asociación de Comerciantes e Industriales del Municipio Lagunillas (ACIL). Siendo los sujetos informantes los gerentes (Gte) y administradores (Adres). En el cuadro 1 se muestra la población asociada al estudio.

Cuadro 1. Población asociada al estudio.

\begin{tabular}{lcc}
\hline \multicolumn{1}{c}{ FERRETERAS } & GTE & ADRES \\
\hline Ferretería Eléctrica Industrial, C.A. & 2 & 2 \\
Distribuidora Hermanos Rodríguez Hernández C.A & 2 & 1 \\
Ferretería HD Miranda C.A & 2 & 2 \\
Ferretería Zulia C.A. & 3 & 2 \\
Ferretería Bolívar S.A. & 3 & 2 \\
Ferretería Comercial LADA C.A. & 4 & 3 \\
Tamayo C.A. & 3 & 2 \\
Sub-Total & $\mathbf{1 9}$ & $\mathbf{1 4}$ \\
Total & & $\mathbf{3 3}$ \\
\hline
\end{tabular}

Fuente: Elaboración propia (2020)

Se utilizó la técnica de la encuesta, y como instrumento un cuestionario, diseñado con tres (03) alternativas de respuestas: Siempre (3), a veces (2) y nunca (1) el mismo constó de 18 ítems. Para la validación del mismo, fue sometido a un proceso de revisión por parte de cinco (5) jueces expertos, los cuales midieron la correspondencia del mismo en su contexto teórico y metodológico. Posteriormente, para el cálculo de la confiabilidad del instrumento se utilizó el método coeficiente Alfa de Crombach, el cual fue de 0,862. 
Para el análisis estadístico, se utilizó la ítems e indicadores, tomando como punto de técnica de la estadística descriptiva, a través de interés de acuerdo a lo que se pretendía medir la media como medida de tendencia central el nivel de presencia, lo cual determinaría si más utilizada, se establece el baremo para la interpretación de la media aritmética de los eran fortalezas o debilidades de la gestión, como se visualiza en el cuadro 2.

Cuadro 2. Baremo para la interpretación de la media aritmética

\begin{tabular}{ccl}
\hline RANGO & CATEGORÍA & DESCRIPCIÓN \\
\hline $2.35-3.00$ & Alta presencia & $\begin{array}{l}\text { Si la presencia es alta, se consideraba una fortaleza para la gestión de } \\
\text { inventario. }\end{array}$ \\
$1.68-2.34$ & Mediana presencia & $\begin{array}{l}\text { Si la presencia es mediana o baja, se consideraba una debilidad para } \\
\text { la gestión de inventario, por tanto requiere mejoras. }\end{array}$ \\
$1-1.67$ & Baja presencia & \\
\hline
\end{tabular}

Fuente: Elaboración propia (2020)

RESULTADOS Y DISCUSION

En la tabla 1, se resumen los resultados alcanzados para el evento de estudio, evidenciando una media de 2,72, implicando alta presencia de los elementos de apoyo a la gestión de inventario que realizan las pequeñas y medianas empresas ferreteras del municipio Lagunillas, delineándola como fortalezas de esta gestión.

Tabla 1. Elementos de apoyo a la gestión de inventario

\begin{tabular}{lcc}
\hline \multicolumn{1}{c}{ Indicadores } & Media & Categoría \\
\hline Infraestructura & 2,95 & Alta presencia/Fortaleza \\
Talento humano & 2,97 & Alta presencia/Fortaleza \\
Proveedor & 2,79 & Alta presencia/Fortaleza \\
Capacitación del personal & 2,26 & Mediana presencia/Debilidad \\
Innovación tecnológica & 2,45 & Alta presencia/Fortaleza \\
Delegación de responsabilidades & 2,80 & Alta presencia/Fortaleza \\
Plazo de entrega & 2,52 & Alta presencia/Fortaleza \\
Disponibilidad & 2,82 & Alta presencia/Fortaleza \\
Transporte & 2,91 & Alta presencia/Fortaleza \\
Evento & $\mathbf{2 , 7 2}$ & Alta presencia/Fortaleza \\
\hline
\end{tabular}

Fuente: Elaboración propia (2020) 
De manera específica, se pudo evidenciar alta presencia de la gestión de inventario que estas empresas ejecutan en relación a todos los indicadores utilizados para medir los elementos de apoyo de la misma, siendo considerados fortalezas de la gestión llevada a cabo; a excepción de la capacitación de personal la cual se catalogó con mediana presencia siendo esto una debilidad de la gestión e indicando que la misma requiere mejoras al respecto.

Estos resultados evidenciados tienen alta congruencia con Silva (2006), cuando afirma, la gestión de inventario posee un conjunto de elementos de apoyo a ella involucrados, que permiten la efectividad de las actividades planificadas, logrando mantener un control acerca de los bienes que dispone la organización, y logrando establecer una continuidad en sus procesos para alcanzar los objetivos establecidos. Donde Render y col. (2006) afirman, existen varios elementos de apoyo a la gestión de inventario, entre estos: infraestructura, talento humano, proveedor, capacitación del personal, innovación tecnológica, delegación de responsabilidades, entrega, disponibilidad y transporte.

Lo mostrado permite al investigador afirmar que, en la gestión de inventario de las empresas analizadas, se posee alto nivel de reconocimiento de la importancia que debe dársele a los elementos de apoyo a esta gestión, a través de la alta presencia de dichos elementos. De tal forma, tienen presente que los elementos de apoyo a la gestión de inventario son todos los elementos presentes en la organización que le permiten establecer sus debilidades y fortalezas para el desarrollo de cualquiera de las gestiones realizadas, en este caso la de inventario.

En lo concerniente al detalle de cada uno de los indicadores utilizados, para medir el evento, se especifican los resultados obtenidos al respecto, mostrados en la tabla 2. De esta forma, se evidencia alta presencia para todos los indicadores - elementos medidos, a excepción de la capacitación del personal el cual fue catalogado con mediana presencia con una media de 2,26; siendo esto una debilidad para la gestión.

En específico, otorgaron mediana presencia a las actividades medidas en este indicador referidas a veces el personal recibe adiestramiento en el manejo de los diferentes sistemas de información de inventario que posee la empresa $(2,33)$; y a veces realizan cursos que permitan desarrollar tanto sus competencias genéricas como técnicas en materia de inventario $(2,18)$, señalándolas como debilidades.

El resto de los indicadores muestran medias aritméticas que los posicionan en alta presencia, siendo fortalezas de la gestión: infraestructura $(2,95)$; talento humano $(2,97)$; proveedor $(2,79)$; innovación tecnológica $(2,45)$; delegación de responsabilidades $(2,80)$; plazo de entrega $(2,52)$; disponibilidad $(2,82)$; y transporte $(2,91)$.

Desglosando cada uno de los indicadores por ítem, se conoció que todos ellos arriban a la categoría de alta presencia, ubicándolos como fortalezas de la gestión; exceptuando los ítems del indicador capacitación del personal (ya mencionados) y el ítem 9 del indicador innovación tecnológica valorados con mediana presencia, por ende son debilidades de la gestión. En cuanto al ítem 31 , se le consultaba a los encuestados a veces ejecutan cursos de formación que permitan el conocimiento de las nuevas tecnologías para el manejo de inventarios $(2,33)$, considerándose una debilidad para la gestión de inventario estudiada, por tanto requiere mejoras según el baremo utilizado. 
Tabla 2. Indicadores del evento: Elementos de apoyo a la gestión de inventario

\section{Indicador: Infraestructura}

Ítems

1. Cuentan con instalaciones que permitan la realización efectiva de las tareas relacionadas al inventario de materiales.

2. Poseen los recursos en infraestructura necesarios para llevar a cabo 2,91 la ejecución de sus actividades operacionales.

\section{Promedio Indicador 2,95}

\section{Indicador: Talento humano}

\section{Ítems}

3. Cuentan con personal calificado para el desarrollo de los diferentes procesos de inventario, impactando en la productividad generando un valor agregado.

4. El personal posee los conocimientos necesarios que le permitan llevar a cabo sus actividades, brindando aportes estratégicos a los procesos de gestión de inventario.

\section{Promedio Indicador 2,97}

\section{Indicador: Proveedor}

\section{Ítems}

5. Cuenta con una cartera de proveedores certificados para los 2,82 diferentes tipos de materiales requeridos.

6. El nivel de cumplimiento de los proveedores les permite mantener 2,76 abastecidos los almacenes con los diferentes productos demandados por los clientes.

\section{Media}

Media

Media

3,00

Alta presencia Fortaleza

\section{Alta presencia} Fortaleza

\section{Categoría}

Alta presencia

Fortaleza

Alta presencia

Fortaleza

Alta presencia Fortaleza 
Tabla 2. Indicadores de la dimensión: Elementos de apoyo a la gestión de inventario (continuación)

\section{Indicador: Capacitación del personal}

\section{Ítems}

7. El personal recibe adiestramiento en el manejo de los diferentes sistemas de información de inventario que posee la empresa.

8. El personal realiza cursos que permitan desarrollar tanto sus 2,18 competencias genéricas como técnicas en materia de inventario.

Promedio Indicador 2,26

\section{Indicador: Innovación tecnológica}

\section{Ítems}

9. Ejecutan cursos de formación que permitan el conocimiento de las nuevas tecnologías para el manejo de inventarios.

10. Gestionan con mayor fuerza la tecnología para ofrecer un mejor servicio al cliente.

Promedio Indicador $\mathbf{2 , 4 5}$

\section{Indicador: Delegación de responsabilidades}

\section{Ítems}

11. El gerente delega parte de sus responsabilidades operativas a su equipo de trabajo evitando la concentración de tareas en una sola persona.

12. El gerente posee la capacidad de delegar efectivamente responsabilidades, evitando dedicar esfuerzo o tiempo a tareas que otro puede realizar con eficiencia.

\section{Media Categoría}

2,33 Mediana presencia

Debilidad

Mediana presencia

Debilidad

\section{Mediana presencia}

Debilidad

\section{Media}

2,33

\section{Categoría}

Mediana presencia

Debilidad

2,58

Alta presencia

Fortaleza

\section{Alta presencia \\ Fortaleza}

Media

2,76

Categoría

Alta presencia

Fortaleza

\section{2,85 Alta presencia}

Fortaleza

\section{Alta presencia \\ Fortaleza}


Tabla 2. Indicadores de la dimensión: Elementos de apoyo a la gestión de inventario (continuación)

\section{Indicador: Plazo de entrega}

\section{Ítems}

13. La ubicación geográfica de los proveedores limita el cumplimiento en la entrega de los materiales.

14. Emiten la orden de pedido en el tiempo establecido garantizando la entrega oportuna de los materiales por parte del proveedor.

\section{Promedio Indicador 2,52}

\section{Indicador: Disponibilidad}

\section{Ítems}

15. La entrega de los materiales por parte de los proveedores garantiza la ejecución de las ventas en el tiempo estimado.

16. Cuentan con un inventario de materiales que cumpla con los requerimientos exigidos por los clientes.

\section{Promedio Indicador 2,82}

\section{Indicador: Transporte}

\section{Ítems}

17. Disponen de unidades de movilización de materiales desde el almacén hasta el sitio de utilización garantizando las actividades comerciales.

18. Cuenta con la logística necesaria para trasladar los materiales de importancia en el momento preciso.
Media

2,39

2,64

Alta presencia

Fortaleza

Alta presencia

Fortaleza

\section{Media}

2,67

2,97

Alta presencia

Fortaleza

Alta presencia

Fortaleza

Alta presencia

Fortaleza

\section{Media Categoría}

2,91 Alta presencia

Fortaleza

Alta presencia

Fortaleza

Alta presencia

Fortaleza

Alta presencia

Fortaleza

Fuente: Elaboración propia (2020) 
Lo mostrado permite afirmar, en la gestión de inventario de las empresas analizadas se han asumido los elementos de apoyo a su gestión como una logística coordinada, encargada de minimizar los recursos financieros invertidos en la gestión de inventario. No obstante, deben revisar las actividades encaminadas a la capacitación del personal pues han sido vistas con mediana presencia, así como lo relacionado a la actividad involucrada a la innovación tecnológica, en ambos casos ya señaladas, pues están siendo catalogadas como debilidades de su gestión.

En este aspecto, a juicio del investigador, en las empresas analizadas, se tiene claro el rol que deben jugar estos elementos para el logro de una efectiva gestión de inventario, acatando que se han identificado estos elementos como lo plantea Ballou (2004), para quien los elementos de apoyo a la gestión de inventario permiten a la empresa decidir los medios a necesitar para sus operaciones y distribuir esos medios fabricar el producto deseado en las cantidades requeridas, en el menor tiempo y costo posible.

Por lo obtenido, estos elementos se desarrollan en las empresas apoyando la gestión de inventario sobre la base de una planificación exhaustiva considerando el desempeño de los procesos organizacionales, para disponer de materias primas y demás elementos de fabricación en el momento oportuno y lugar requerido; reducir períodos muertos de maquinaria y obreros; asegurar que éstos no trabajan en exceso ni estén inactivos.

\section{CONCLUSIONES}

La información recolectada, en las pequeñas y medianas empresas ferreteras del municipio Lagunillas, referida a los elementos de apoyo a la gestión de inventario, se detectó alta presencia en conjunto de estos elementos, y de manera detallada alta presencia en ocho (8) de nueve (9) elementos medidos, donde la capacitación del personal se catalogó con mediana presencia, acotando debilidades en actividades referidas a este elemento.

De manera concreta, las debilidades encontradas se centran en si el personal recibe adiestramiento en el manejo de los diferentes sistemas de información de inventario que posee la empresa y si realizan cursos que permitan desarrollar tanto sus competencias genéricas como técnicas en materia de inventario. También existe una debilidad en el elemento innovación tecnológica, en lo concerniente a si ejecutan cursos de formación que permitan el conocimiento de las nuevas tecnologías para el manejo de inventarios.

Siendo así, se puede concluir que los elementos de apoyo a la gestión de inventario es una fortaleza para las empresas analizadas, en específico en cuanto a infraestructura, talento humano, proveedor, innovación tecnológica, delegación de responsabilidades, plazo de entrega, disponibilidad y transporte. No obstante, se evidencian oportunidades de mejora en lo concerniente a la capacitación del personal y en el elemento innovación tecnológica. Por otro lado, permiten establecer sus debilidades y fortalezas para el desarrollo de cualquiera de las gestiones realizadas en este caso la de inventario. Todos estos elementos, en estas empresas, se interrelacionan con una logística coordinada, encargada de minimizar los recursos financieros invertidos en la gestión de inventario. 
REFERENCIAS BIBLIOGRAFICAS

Agueda, E. García, J. Narros, M. Olarte, C. Reinares, M. y Saco, M. (2008). Principios de Marketing. Tercera Edición. ESIC Editorial. Madrid España

Alles, M. (2005). Desarrollo del talento humano. Basado en competencias. Primera Edición. Ediciones Granica. Buenos aires. Argentina

Alles, M. (2007). Rol del jefe. Como ser un buen jefe. Primera Edición. Ediciones Granica. Buenos aires. Argentina

Anaya, J. (2014). El diagnóstico logístico: Una metodología para promover mejoras competitivas. Libros profesionales de empresa. Libros profesionales de empresa. Editorial ESIC. Madrid. España

Ballou, R. (2004). Logística. Administración de la cadena de suministro. Quinta Edición. Editorial Pearson Prentice Hall. México

Bowersox, D. Colss, D. y Cooper, B. (2007). Administración y Logística en la cadena de suministros. Editorial McGrawHill/Interamericana Editores, S.A. de C.V. México

Escorsa, P. y Valls, J. (2005). Tecnología e innovación de la empresa. Segunda Edición. Alpha - Omega Grupo Editores, S.A. Distrito Federal, México

Escudero, J. (2011). Gestión de aprovisionamiento. Tercera Edición. Ediciones paraninfo, S.A. Madrid España

Fernández, E. (2010). Administración de empresas un enfoque interdisciplinar. Primera Edición. Madrid España
García, A. (2008). Enfoques prácticos para planeación y control de inventarios. Cuarta Edición. Editorial Trillas. México

Ivancevich, J., Konopaske, R., Matteson M. y Núñez, J. (2010) Comportamiento organizacional y administración de empresas. Décima edición. Editorial McGraw-Hill Interamericana de España S.L. España

Marcano, P. (2011). Gestión de producción y su competitividad en empresas venezolanas. Revista de Ciencias Sociales, Vol. 1, № 1. Universidad Nacional Experimental Rafael María Baralt. Cabimas. Venezuela

Render, B. Stair, R. y Hanna, M. (2006). Métodos cuantitativos para los negocios. Novena Edición. Editorial Pearson Educación. México

Rozas, P. y Ricardo, S, (2005). Desarrollo de infraestructura y crecimiento económico: revisión conceptual. Santiago de Chile. Chile

Silva, G. (2006). La gestión de operaciones competitiva. Editorial McGraw-Hill. México

Vollmann, T. Berry, W. Clay, D. y col. (2008). Planeación y Control de la Producción. Administración de la Cadena de Suministros. Quinta Edición. McGraw-Hill. México

Werther, Jr., William B. y Davis, Keith, (2008). Administración de personal y recursos humanos. McGraw-Hill Interamericana. España 